\title{
TbFeCo as a Perpendicular Magnetic Recording Material
}

\author{
Kazuyuki Ozaki, Koji Matsumoto, Ikuya Tagawa*, and Keiji Shono \\ FUJITSU LABORATORIES LTD, Nishiwaki-64, Ohkubo-cho, Akashi 674-8555, Japan \\ * FUJITSU LABORATORIES LTD, 10-1 Morinosato-Wakamiya, Atsugi 243-0197, Japan
}

(Received Dec. 10, 2000; Accepted Dec. 21, 2000)

\begin{abstract}
TbFeCo perpendicular magnetic recording media were developed by employing a NiP underlayer to control the magnetic properties of $\mathrm{TbFeCo}$ magnetic layer. With the NiP underlayer the magnetization reversal changed from the wall motion to the rotation mode. Dynamic write-read characteristics of a single-layer medium were examined using a conventional merge-type GMR head with a write core width of $1 \mu \mathrm{m}$ that was designed for longitudinal magnetic recording. A clear magnetic pattern of $450 \mathrm{kFCl}$, high media SNR and acceptable overwrite properties were obtained. It was shown the thermal stability of the $\mathrm{TbFeCo}$ medium is good enough for practical applications. A double-layer medium with a soft-magnetic backlayer exhibited a practical overwritability for a narrow track pitch of $0.4 \mu \mathrm{m}$.
\end{abstract}

Key words: magneto-optical recording media, amorphous RE-TM alloy film, TbFeCo, perpendicular magnetic recording, GMR head, merge type head

\section{Introduction}

Co-Cr based alloy films have been investigated as materials for perpendicular recording media. The films have a structure composed of segregated grains and the main media noise source is in-bit reversed magnetic domain "). To decrease the noise, both of the reversed domain formation and the reversed domain size must be reduced. Honda et al. ${ }^{2)}$ showed that the squareness ratio $(\mathrm{Mr} / \mathrm{Ms}=\mathrm{S})$ in the perpendicular $\mathrm{M}-\mathrm{H}$ loop must be increased to suppress the reversed domain formation, and the magnetic exchanging coupling between adjacent grains must be reduced to reduce the reversed domain size. The smaller exchange coupling leads to a smaller loop slope in $\mathrm{M}-\mathrm{H}$ loop. Large $\mathrm{S}$ is also required to prevent thermal fluctuation of perpendicular recording media at lower linear density due to higher demagnetization field ${ }^{3)}$. However, larger $\mathrm{S}$ and smaller loop slope are in a tradeoff relationship and very high coersivity $(\mathrm{Hc})$ is required to satisfy both of them for an appropriate Ms value, which makes it difficult to fabricate media.

On the other hand, Rare Earth - Transition Metal (RE-TM) alloy films that are widely used as magnetooptical (MO) recording materials have an amorphous and continuous structure. Recently new recording technologies combining thermo-magnetic or thermally assisted magnetic writing on MO media and magnetic flux reading by GMR sensor were proposed to achieve higher density recording ${ }^{4), 5}$.

From these backgrounds and seeking another approach, it seems a good way to apply RE-TM alloy films for perpendicular magnetic recording media because reversed domains formation can be suppressed according to their high squareness ratio and low media noise is expected according to their amorphous continuous structure. In addition, they have features preferable to magnetic recording such as; a rather large perpendicular magnetic anisotropy, $\mathrm{Ku}$, easily controllable magnetic properties and fabrication at room temperature. Figure 1 shows the dependence of $\mathrm{Ku}$ and $\mathrm{Ms}$ of $\mathrm{TbFe}$ film on the composition for example, which are calculated using the molecular field model. Because of the characteristics of ferrimagnetic material, there exists the composition of zero net magnetization, which is called the compensation composition. This means that $\mathrm{Ms}$ is tunable over a wide range by composition. The value of $\mathrm{Ku}$ is $3.5-5 \times 10^{6}$ $\mathrm{erg} / \mathrm{cm}^{3}$, which is larger than that of $\mathrm{Co}-\mathrm{Cr}$ alloy film (about $2 \times 10^{6} \mathrm{erg} / \mathrm{cm}^{3}$ at the maximum).

Indeed, investigations have already been done about perpendicular magnetic recording on RE-TM alloy films. Jenniot et al. ${ }^{6)}$ and Berstein et al. ${ }^{7)}$ demonstrated perpendicular recording on $\mathrm{TbGdFe}, \mathrm{TbFe}$, and $\mathrm{GdFe}$ thin films. Ohtani et al. examined the relationship between film fabrication conditions and read-write properties ${ }^{8}$. However, desirable recording characteristics were not obtained in any experiments and amorphous RE-TM media with strong exchange interaction in film have been believed to be unsuitable for magnetic recording because

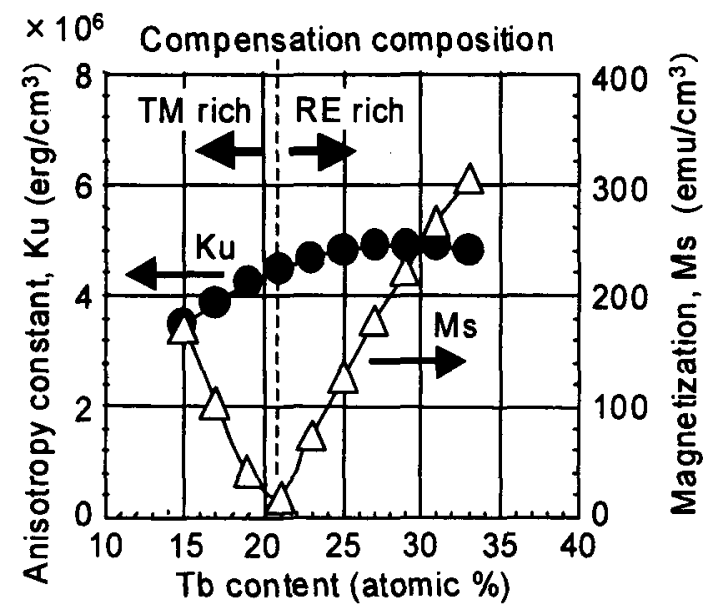

Fig. 1 Composition dependence of anisotropy constant, $\mathrm{Ku}$, and saturation magnetization, $\mathrm{Ms}$, of TbFe calculated using the molecular field model. 
of the distortion of magnetic transition induced by domain wall motion. However, we think issues in recording performance might have also caused these results. For example, a sensitive GMR reading head was not yet available, and the sputtering technique was not sophisticated at that time.

In this paper, we demonstrate the capabilities of high recording density on perpendicular magnetic recording media composed of a TbFeCo film by controlling the magnetization reversal mode and improving a sputtering process. Dynamic write-read characteristics of a singlelayer medium were examined using a conventional merge type GMR head designed for longitudinal recording media. A double-layer medium with a soft-magnetic backlayer was also evaluated using a head with a narrow core width.

\section{Preparation of single-layer media}

\subsection{Structure and basic properties}

Single-layer perpendicular media with the structure shown in Fig. 2 were fabricated by a conventional DC magnetron sputtering at room temperature on 2.5 inch size glass substrates. A thin SiN layer was formed on the glass substrate as an adhesive layer, and on top of that, a NiP underlayer with a thickness of $10 \mathrm{~nm}$ was sputtered. Then, 40nm thick TbFeCo with TM-rich composition was sandwiched between protection layers of amorphous carbon. A lubricant was spin-coated on the top carbon layer for dynamic write-read measurements. Figure 3 shows the Kerr rotation hysteresis loop. The medium has a squareness ratio of 1 and a coercivity squareness $S^{*}$ of over 0.7 without compensation for demagnetizing field. The $\mathrm{Hc}$ of the samples are $2.9 \mathrm{kOe}$, and the product of film thickness and saturation magnetization, tMs, is about $1.0 \mathrm{memu} / \mathrm{cm}^{2}$.

\subsection{Effect of NiP underlayer}

The NiP underlayer plays an important role to control the magnetic properties of the media ${ }^{9)}$. In order to investigate the magnetization reversal mode, the angular dependence of $\mathrm{Hc}$ of $\mathrm{TbFeCo}$ films with a NiP underlayer and without a NiP underlayer were measured. As shown in Fig. 4, the Hc of a film with a NiP underlayer decreases with varying magnetic field direction from normal ( 0 degree) to parallel (90 degree) to a film plane, but the Hc of a film without a NiP underlayer increases with changing magnetic field direction on the contrary. The result suggests that the magnetization of TbFeCo film without a NiP underlayer reverses by the domain wall movement mode, but the magnetization of $\mathrm{TbFeCo}$ film with a NiP underlayer reverses mainly by the rotation $\operatorname{mode}^{(0)}$.

Figure 5 shows the morphology of a media surface observed with an atomic force microscope. Without NiP, the surface roughness is $0.2 \mathrm{~nm}$, but with the NiP underlayer, the roughness is $0.3 \mathrm{~nm}$. Figure 6 shows the effect of the NiP underlayer on the shape of perpendicular

\begin{tabular}{|c|c|}
\hline Lubricant & \\
\hline Protection layer $(10 \mathrm{~nm})$ & Carbon \\
\hline Recording layer (40 nm) & $\mathrm{TbFeCc}$ \\
\hline Protection layer $(5 \mathrm{~nm})$ & Carbon \\
\hline 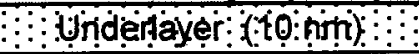 & NiP \\
\hline Adhesive layer $(10 \mathrm{~nm})$ & $\mathrm{SiN}$ \\
\hline Substrate & Glass \\
\hline
\end{tabular}

Fig. 2. Structure of the TbFeCo single-layer perpendicular recording media

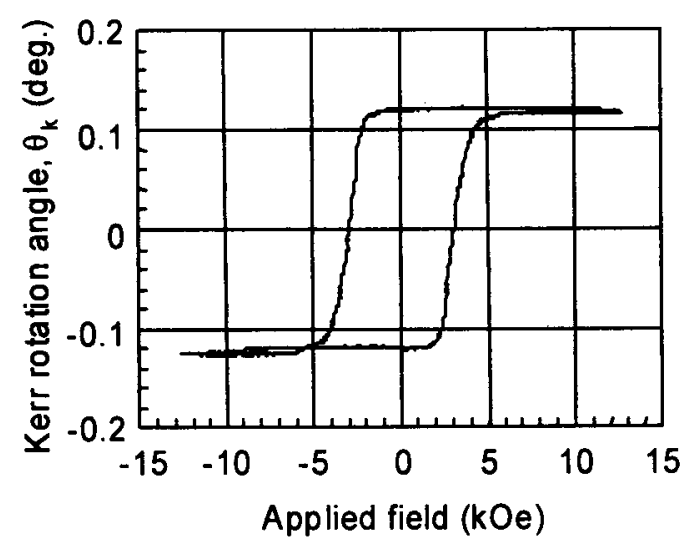

Fig. 3. Kerr rotation hysteresis loop.

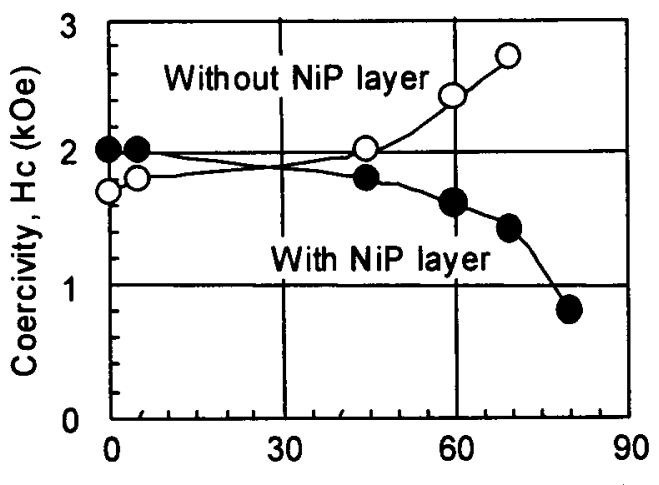

Field angle from film normal (deg.)

Fig. 4. Angular variation of Hc for the samples with and without NiP umderlayer.

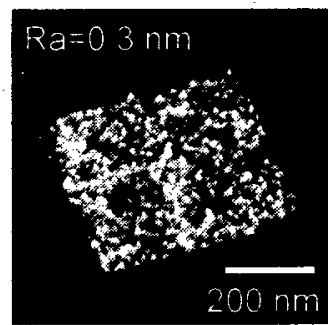

(a) With NiP layer

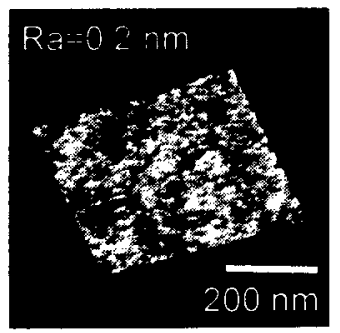

(b) Without NiP layer
Fig.5. Surface morphology of the samples (a) with and (b) without NiP umderlayer. 


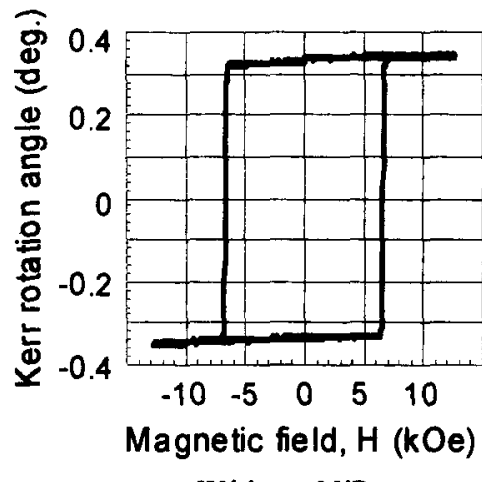

W ithout NiP

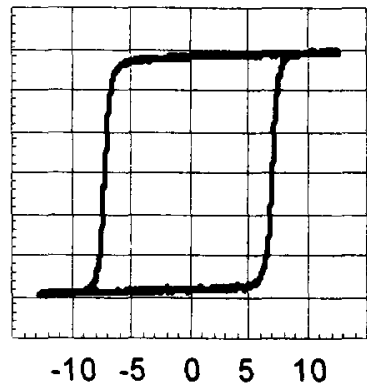

Magnetic field, $\mathrm{H}(\mathrm{kOe})$

$8 \mathrm{~nm} \mathrm{NiP}$

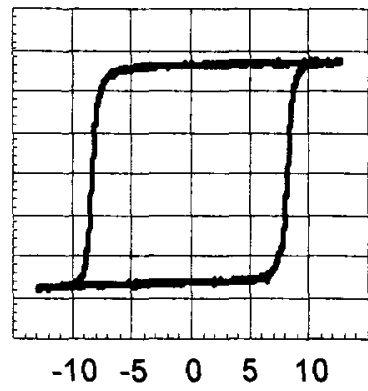

Magnetic field, $\mathrm{H}$ (kOe)

$16 \mathrm{~nm} \mathrm{NiP}$

Fig.6. Effect of NiP underlayer on the shape of hysteresis loop

hysteresis loop. Without NiP underlayer the slope of the loop is very steep. With NiP the slope of the loop decreases and the coercive force increases as the thickness increases, while the squareness ratio remains 1 . We believe the difference in the surface morphology of the bottom carbon layer brought about by the NiP layer introduces wall motion pinning sites in the TbFeCo film, which changes the magnetization reversal mode.

\section{Evaluation results of single-layer media}

Dynamic properties of the single-layer medium were measured with a spin stand tester equipped a merge type GMR head (write core width: $1.0 \mu \mathrm{m}$, write gap length: $0.3 \mu \mathrm{m}$, read core width: $0.8 \mu \mathrm{m}$, read gap length: 0.12 $\mu \mathrm{m})$ designed for longitudinal recording media. The linear velocity at writing and reading was $12 \mathrm{~m} / \mathrm{s}$.

\subsection{Magnetic transitions}

Figure 7 shows the magnetic force microscope images of the written magnetic patterns on the TbFeCo medium. Reversed domains, which are often observed on $\mathrm{Co}-\mathrm{Cr}$ based alloy perpendicular recording media, do not take place inside the written domains even for long marks (53kFCI). The distortion of magnetic transitions is very small and clear magnetic patterns can be observed even at $450 \mathrm{kFCI}$, although the edge of the write head has a "percolation.". This indicates that high linear density recording is possible, even on films having rather large exchange couplings

\subsection{Dynamic properties}

Figure 8 (a) shows the reproduced waveform of an isolated pattern, which has a shape typical of single-layer perpendicular media. The dipulse ratio is 1 , which means that recording is free of demagnetization at the trailing edge of the write head because the media has a high coercivity squareness. Figure 8 (b) shows the waveform of a random pattern. The waveform of $330 \mathrm{kFCI}$ is clearly resolved. We observed a signal even at $650 \mathrm{kFCI}$.

Figure 9 shows the media signal-to-noise ratio, media SNR, and Fig. 10 shows the peak-to-peak signal amplitude, $\mathrm{S}_{\mathrm{PP}}$, of the $\mathrm{TbFeCo}$ medium and the longitudinal medium with a capability of $15 \mathrm{~Gb} / \mathrm{in}^{2}$. The

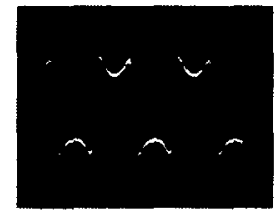

(a)

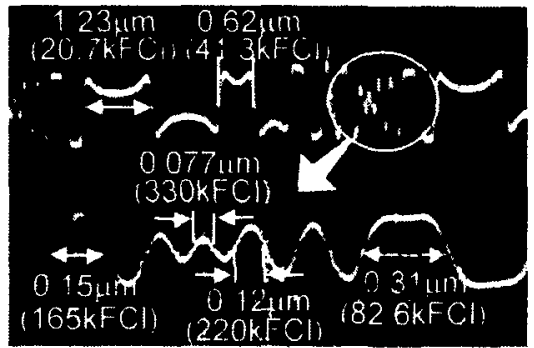

(b)
Fig. 8 The reproduced waveforms of (a)isolated pattern and (b)random pattern.
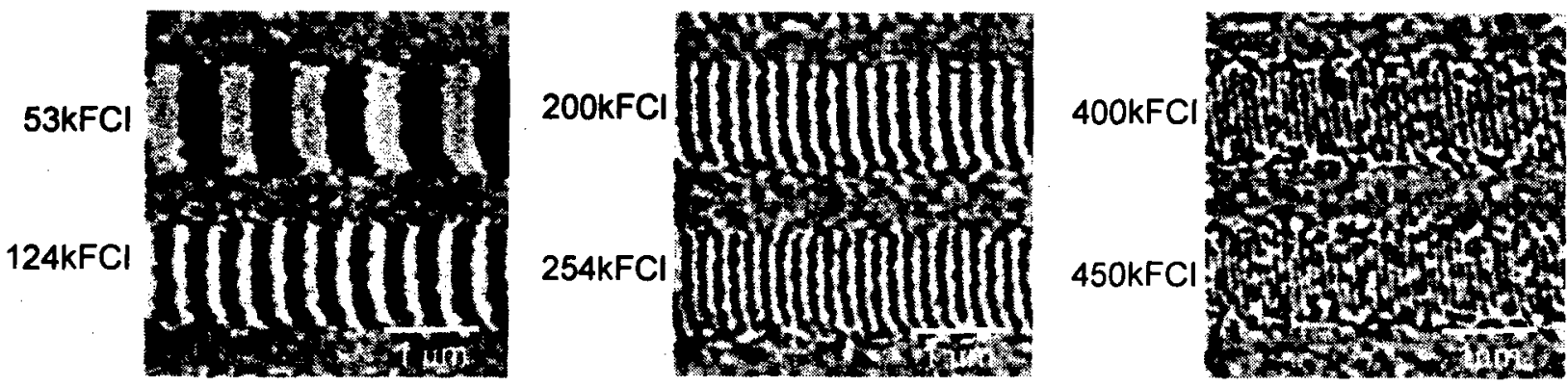

Fig. 7 Magnetic force microscope images of magnetic patterns recorded by a ring-type write head with a core width of $1.0 \mu \mathrm{m}$ and a gap length of $0.3 \mu \mathrm{m}$. 
media $\mathrm{SNR}$ is defined as $\mathrm{SNR}=20 \log _{10}\left(\mathrm{~S}_{\mathrm{PP}} / \mathrm{N}_{\mathrm{rms}}\right)$, in which $\mathrm{N}_{\mathrm{ms}}$ denotes the media noise that was derived by subtracting system noise and head noise from total noise. Even though media SNR of TbFeCo media is smaller than that of longitudinal media, total SNR including system noise and head noise is not different in each media because we used $\mathrm{TbFeCo}$ media with a large signal amplitude.

Figure 11 shows the dependence of signal amplitude and overwrite properties on a write current, Iw. The signal

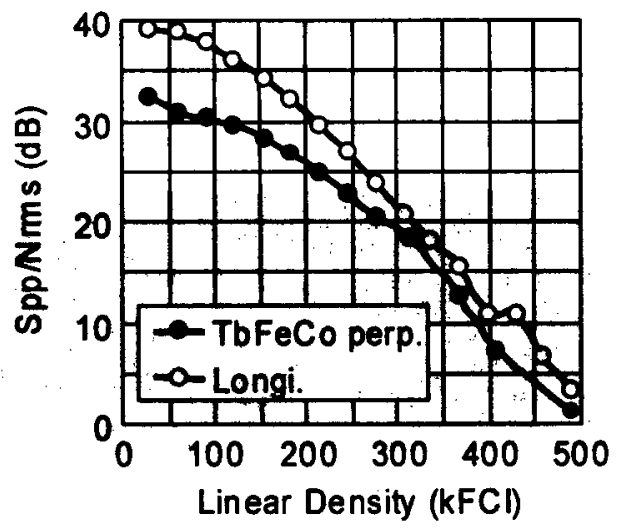

Fig. 9 The linear density dependence of media signal to noise ratio.

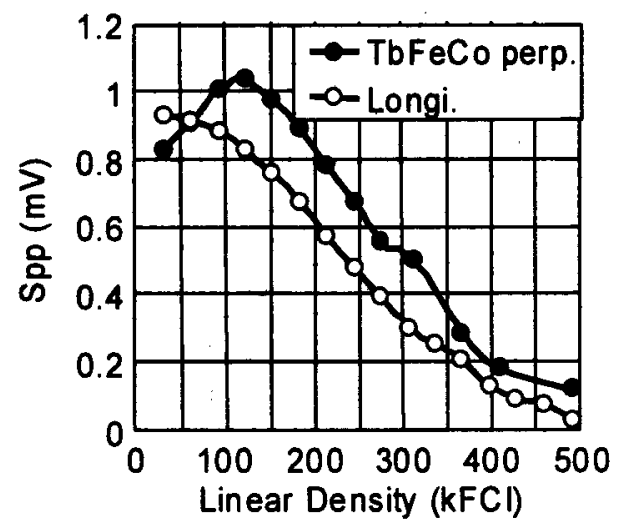

Fig. 10 The linear density dependence of signal amplitude.

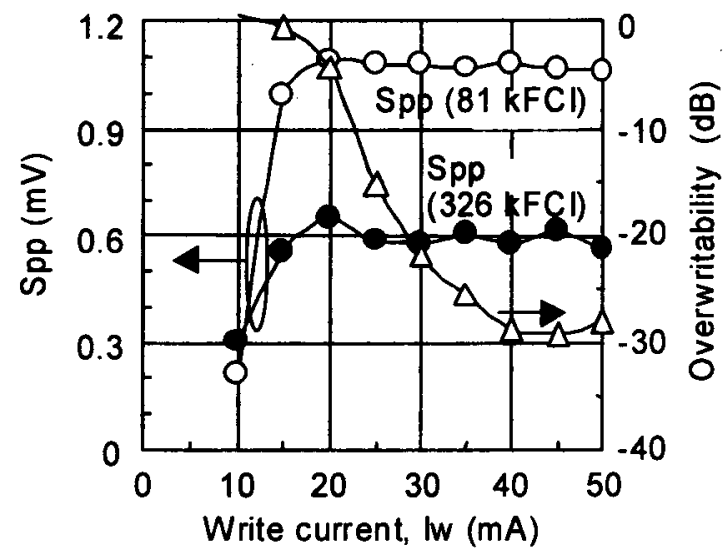

Fig. 11. Write current dependence of signal amplitude and overwritability. amplitude was measured for $81 \mathrm{kFCI}$ and $326 \mathrm{kFCI}$. Without recording demagnetization, the amplitude became saturated as Iw increased. Overwrite properties were examined by measuring the residual signal level of 81 kFCI after overwriting at $490 \mathrm{kFCI}$. The overwritability value is $-30 \mathrm{~dB}$, which is at an acceptable level for practical use.

\subsection{Thermal stability}

Static thermal stability of the TbFeCo medium was measured. A sample of the medium was saturated, and the temporal change in residual magnetization $\mathrm{Mr}$ without applying a magnetic field was measured using SQUID. Figure 12 shows the change of $\mathrm{Mr} / \mathrm{Ms}$ against time at $25^{\circ} \mathrm{C}$ and $65^{\circ} \mathrm{C}$. Mr/Ms has no decay at $25^{\circ} \mathrm{C}$, even after $10^{4}$ seconds have elapsed. But at a high temperature of $65^{\circ} \mathrm{C}, \mathrm{Mr} / \mathrm{Ms}$ decreased at a rate of $1.3 \% /$ decade because of the reduction of $\mathrm{Hc}$. This rate is not so big, and moreover this would be improved by using high-Curiepoint media or RE-rich media

The changes of recorded signal output voltage were also measured against time using a spin stand tester for 42 $\mathrm{kFCI}, 200 \mathrm{kFCI}$, and $400 \mathrm{kFCI}$ at $25^{\circ} \mathrm{C}$. It is known that perpendicular recording is subject to thermal agitation at lower linear density because of higher demagnetization field. As shown in Fig. 13, all linear densities did not have

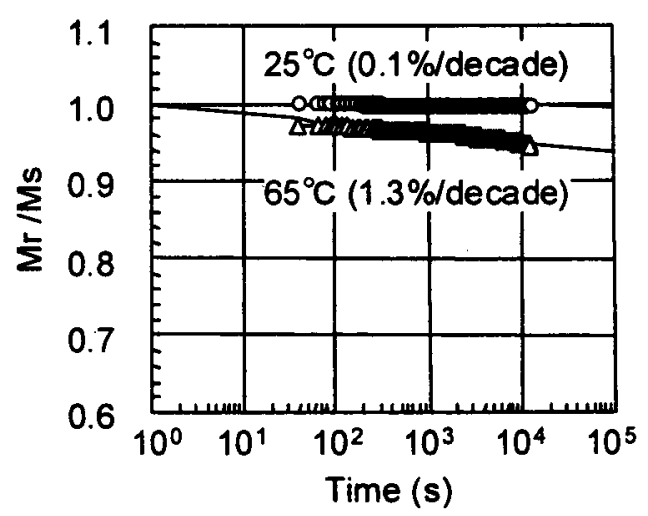

Fig. 12 Time dependence of $\mathrm{Mr} / \mathrm{Ms}$ for the $\mathrm{TbFeCo}$ perpendicular recording media.

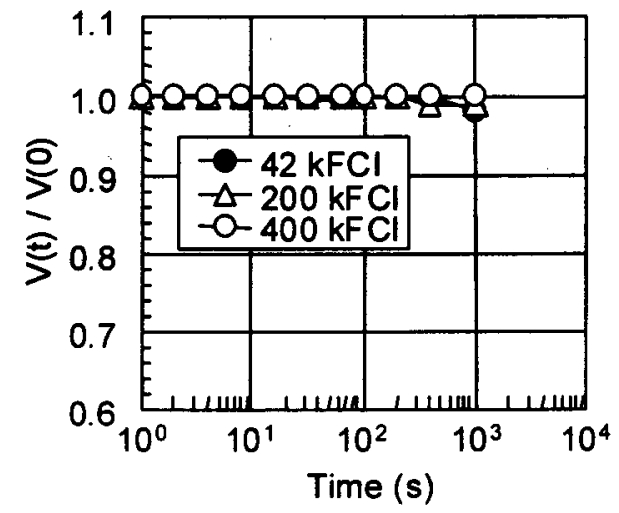

Fig. 13 Time dependence of output signal voltage for different linear densities. 
significant decay although the measurement time was not long enough, which corresponds to the result of Fig. 12.

\section{Double-layer media}

\subsection{Media and head}

Perpendicular magnetic recording media often have a soft magnetic back-layer to increase the perpendicular writing field in the recording layer by in-plane magnetization in the back-layer. We tried the double-layer media configuration to enhance overwrite performance for a narrow track width.

The media structure is shown in Fig. 14. We used a $\mathrm{CoZrNb}$ amorphous film with a thickness of $500 \mathrm{~nm}$ as a soft magnetic back-layer. The layer structure with $\mathrm{TbFeCo}, \mathrm{NiP}$, and $\mathrm{C}$ in the double-layer media is the same as that of the single-layer media explained in section 3.1 and the $\mathrm{Hc}$ of the double-layer medium is $2.9 \mathrm{kOe}$.

Figure 15 shows the bottom-view of the head that we prepared to evaluate the double-layer medium. A mergetype GMR head having a very narrow write track width of $0.4 \mu \mathrm{m}$ was FIB trimmed from the air bearing surface. As shown in the figure, there are two FIB trimmed depressions. One is to make a wide gap length, and another is to get the straight trailing edge. The obtained configuration resembles a single-pole head for perpendicular recording. On the other hand, a nontrimmed head with the write core width of $0.5 \mu \mathrm{m}$ was used to evaluate the single-layer medium described in section 3 for comparison.

\begin{tabular}{|c|c|}
\hline Lubricant & \\
\hline Protection layer $(10 \mathrm{~nm})$ & Carbon \\
\hline$\because$ Recording layer $(40 \mathrm{~nm}):$ & TbFeCo \\
\hline Protection layer $(5 \mathrm{~nm})$ & Carbon \\
\hline 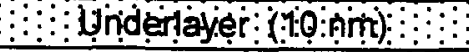 & \\
\hline Soft magnetic layer $(500 \mathrm{~nm})$ & CoZrN \\
\hline Substrate & Glass \\
\hline
\end{tabular}

Fig. 14 Structure of the TbFeCo double-layer media with a soft magnetic back layer.

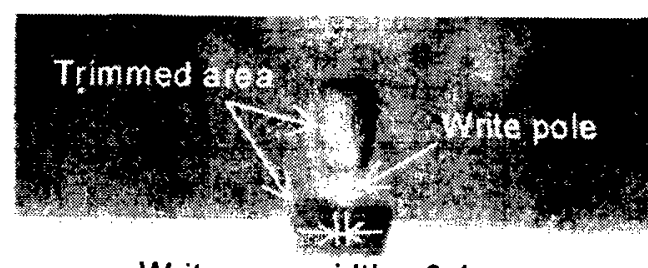

Write core width $0.4 \mu \mathrm{m}$

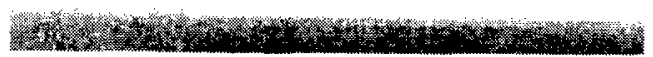

Fig. 15 Bottom-view of the FIB trimmed head for evaluation of the double-layer media

\subsection{Dynamic properties}

Figure 16 is the readout waveforms of the doublelayer medium, which shows the typical shape of perpendicular recording using a single pole head. Overwrite properties of each medium were examined using the residual signal of $81 \mathrm{kFCI}$ after overwriting at $490 \mathrm{kFCI}$ as shown in Fig. 17. Overwrite performance of the single-layer medium is poor for the narrow track width. Conversely, overwritability of the double-layer medium, even for $0.4 \mu \mathrm{m}$ track width, is good enough for practical applications. Figure 18 shows the media SNRs of each medium. Unfortunately, the media SNR of the double-layer medium is smaller than that of the singlelayer medium, because the write noise of the double-layer

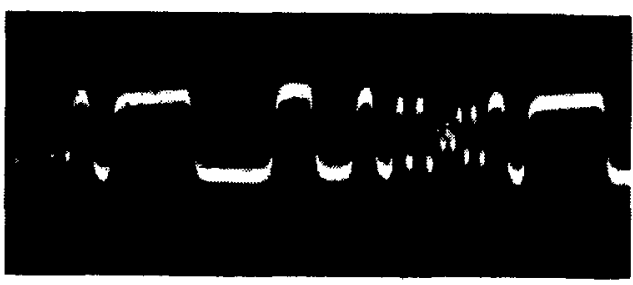

Fig. 16. Reproduced waveforms of the double-layer media.

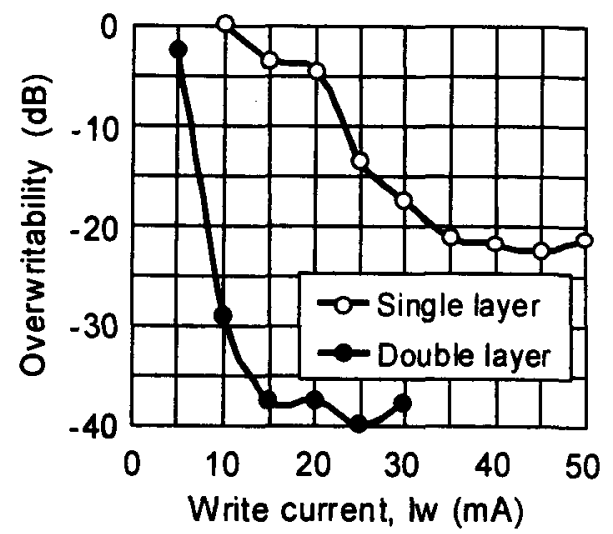

Fig. 17 Write current dependence of overwritability of the double-layer media and the single-layer media for narrow track pitch.

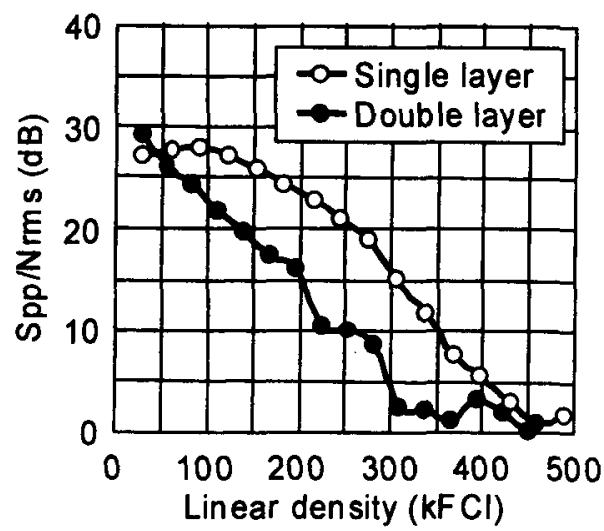

Fig. 18 Media signal to noise ratio of the double-layer media and the single-layer media for narrow track pitch.

J. Magn. Soc. Japan, Vol. 25, No. 3-2, 2001 
medium is rather high. Decrease of the write noise is the next issue for research and the media structure must be optimized for double-layer media.

\section{Conclusion}

We have developed TbFeCo perpendicular magnetic recording media by controlling the magnetic properties. Especially we found that the existence of a NiP underlayer changed the magnetization reversal to the rotation mode, suppressed the distortion of magnetic transition and elevated the recording resolution. The single-layer TbFeCo perpendicular medium exhibited clear magnetic transitions of $450 \mathrm{kFCI}$ and high media SNR using a conventional merge-type GMR head. And the thermal stability of the medium is good enough for practical applications. The double-layer medium with a $\mathrm{CoZrNb}$ soft-magnetic backlayer has practical overwritability for a narrow track pitch of $0.4 \mu \mathrm{m}$, yet the media structure must be improved for double-layer media to achieve a higher media SNR.

\section{References}

1) H. Muraoka, S. Yamamoto, and Y. Nakamura: J. Magn. Magn. Mat., 120, 323 (1993)

2) N. Honda, T. Kiya, and K. Ouchi: J. Magn. Soc. Jpn, 21S2, 505 (1997)

3) N. Honda and K. Ouchi: IEICE Trans. Electron., E80-C, 1180 (1997)

4) H. Saga, H. Nemoto, H. Sukeda, and M. Takahashi: Proc. of Magneto-Optical Recording Int. Symp., Monterey, 1999, J. Magn. Soc. Jpn., 23 (Suppl. No. S1), 225 (1999).

5) H. Katayama, S. Sawamura, Y. Ogimoto, J. Nakajima, K. Kojima, and K. Ohta: Proc. of Magneto-Optical Recording Int. Symp., Monterey, 1999, J. Magn. Soc. Jpn., 23 (Suppl. No. S1), 233 (1999).

6) D. Jenniot and J. Desserre: J. Appl. Phys. 54 (1983) 2820.

7) P. Berstein and F. Rio: IEEE Trans. Magn. Mag-23 (1987) 143.

8) Y. Ohtani, F. Yoshimura and I. Hatakeyama: IEEE Trans. Magn. Mag-26 (1990) 2292.

9) K. Matsumoto, K. Ozaki, A. Chekanov, I. Tagawa, and K. Shono: Jpn. J. Appl. Phys., 39, L1161 (2000).

10) S. Chikazumi: Kyojiseitai no Butsuri (Shokabo, Tokyo, 1984) P.273 [in Japanese]. 